\title{
Developing Emotional Skills and the Therapeutic Alliance in Clients with Alexithymia: Intervention Guidelines
}

\author{
Ana Nunes da Silva \\ Faculdade de Psicologia, Universidade de Lisboa, Lisboa, Portugal
}

\section{Keywords}

Alexithymia - Emotional processing - Emotional regulation . Clinical interventions . Therapeutic relationship.

Psychotherapy

\begin{abstract}
Despite being a longstanding and well-established concept, alexithymia is unfamiliar for many clinicians. This article aimed to address the alexithymia concept from a clinical perspective based on a review of the research on alexithymia intervention. Several strategies are proposed to help clinicians better work with alexithymic clients in psychotherapy. Alexithymia assessment, its impact on the therapeutic alliance, and the difficulties in emotional tasks are highlighted points. Considering alexithymia will inform clinicians' current diagnosis and conceptualization and provide specific targets and venues for intervention, increasing the effectiveness of psychotherapy.

๑) 2021 S. Karger AG, Basel
\end{abstract}

\section{Introduction}

Alexithymia is not a new concept. Sifneos [1] first used it in 1973 to designate a group of cognitive and affective characteristics typical of many clients with somatic disor-

karger@karger.com

(c) 2021 S. Karger AG, Basel

www.karger.com/psp

Karger! ders. Despite being extensively studied, many clinicians are not aware of this concept. Etymologically, alexithymia means "without words for feelings." It derives from Greek and Latin-without ("a") words ("lexus") to emotions ("thymus"). Taylor et al. [2] proposed 3 fundamental elements that defined alexithymia: difficulty identifying feelings, difficulty describing feelings, and externally oriented thinking.

Despite its initial association with psychosomatic disorders, currently, it is identified in several other conditions, such as eating disorders $[3,4]$, substance abuse, and dependency (e.g., [5]), depression [6], personality disorders [7-9], and also autism-spectrum disorders, particularly Asperger' syndrome [10]. Nevertheless, alexithymia is not a fundamental feature of any of these disorders but a related, comorbid condition $[11,12]$ that impacts intervention [13].

Considering that multiple variables are involved in its etiology, a multifactor model best describes alexithymia. Among these are exposure to stressful events, the quality of attachment relationships in childhood, or hereditary factors (e.g., [14-16]). Being a complex construct, it involves not only multiple etiologies but also various maintenance factors. Alexithymia can be a co-occurring condition in several psychological disorders, a way to cope with physical diseases, or even a previous vulnerability for developing psychological disorders (e.g., $[17,18])$. Also, 
it should be better though as a continuous variable or a process rather than a dichotomous or type classification (e.g., [19-21]).

There have been several proposed changes to the conceptualization and measurement of the construct over the years. However, some of these proposals were based on empirical considerations without adequate theoretical justification or were not supported by new clinical observations (for a review see [21]). Also, some older research used alexithymia measures that were subsequently found to have serious psychometric problems, such as the Modified Israel Hospital Psychosomatic questionnaire ( $\mathrm{M}_{-}$ BIQ), the California Q_set Alexithymia Prototype (CAQAP), or the Rorschach alexithymia Scale (RAS) (for a review see [22]). I tried to exclude from this review studies that used those measures. Currently, the most used measures are the Toronto Structured Interview for Alexithymia (TSIA) [23] and the TAS-20 [24], which are presented in the assessment part of these guidelines.

\section{Impact of Alexithymia on Psychotherapy}

Alexithymia has a high prevalence in the clinical population, with $>32.7 \%$ in outpatient psychiatric patients and $47.3 \%$ in inpatients (e.g., $[25,26])$, and it has an important impact on psychotherapy. Several studies have found an association with poor outcomes (e.g., [27, 28]). On the other hand, the possibility of alexithymia being modified by psychotherapy remains a topic of debate (e.g., [13, 29-31]). Studies targeting alexithymia reported significant reductions in its scores following treatment. In contrast, studies that measured changes in alexithymia without employing interventions specifically intended to treat it presented inconsistent results (for a review see [32]).

In general, psychotherapy seems effective for clients with alexithymia. Nevertheless, it is a predictor of residual symptoms after therapy (e.g., [33]), impacts on the therapeutic alliance (e.g., [34-37]), clients present more difficulties in describing what they are feeling, tend to focus on their physical symptoms (e.g., [38]), and difficulty in processing emotions and engaging in emotional tasks [12]. There has been some discussion regarding treatment engagement, considering that alexithymic clients would present more challenges (e.g., [39]). Nevertheless, from the accumulated evidence (e.g., [28]), it is important not to assume that alexithymic clients have reservations about entering or lacking engagement in the treatment. Considering these findings, some attempts have been

Guidelines for Intervention with

Alexithymic Clients made to create or test existing therapeutic models for clients with alexithymia.

Grabe et al. [40] studied an inpatient treatment program including psychodynamic group therapy and found a significant reduction in psychopathology and alexithymia. The duration of the inpatient treatment was between 8 and 12 weeks. Treatment included psychodynamic short-term group psychotherapy (3 times per week; $1.5 \mathrm{~h}$ per session) with an insight-oriented approach, focusing on verbalizing individual emotional and interpersonal problems. Once a week, a group session took place for role plays, including psychodrama. Clients also received an hour per week of individual psychotherapy. Additionally, art therapy, sports therapy, relaxation therapy, and body and movement therapy were offered daily. Considering the positive results, the authors hypothesize that the intensive-care inpatient setting was effective in improving the identification, differentiation, and verbalization of emotions and feelings. These are important aspects when working with alexithymic clients. Nevertheless, they continued to suffer from higher distress at discharge than nonalexithymic individuals.

Levant et al. [41] developed a six-session manualized psychoeducational group intervention (alexithymia reduction treatment [42]). Each session had homework assignments and addressed a central topic: (i) male emotion socialization, (ii) developing a vocabulary for emotions, (iii) learning to read the emotions of others, (iv) keeping an emotional response log, (v) practice, and (vi) moving to deeper issues. Participants in the treatment group demonstrated significant reductions in alexithymia from pretest to post-test compared to the treatment-as-usual group. Two major limitations of this study concern its focus on the male gender and that the findings may be due to the differences in the settings and participants as much as to differences between treatments [41]. Nevertheless, it may suggest the importance of psychoeducation when working with clients with difficulty engaging with their emotions.

Three individual case studies aimed to study a skillbased intervention for the treatment of alexithymia [43] showed the treatment effectively reduced alexithymia. It helped the clients clarify, identify, and describe their feelings. The intervention integrated components that address the relationship between alexithymia and early life experiences, identifying feelings and expressing feelings. Following treatment, participants were more attentive to their emotional states.

In a study guided by an integrative metamodel $[13,36$, 37], a mixed-methods longitudinal design was conducted to understand how emotional processing and change 
process evolved in 12 clients in psychotherapy [13]. Generally, alexithymic clients showed a significant change in psychotherapy. Changes were associated with giving meaning to emotional experience, linking emotions to events, or gaining new perspectives. However, they tended to focus on physical complaints, described changes rationally, and presented vaguer descriptions of their problems than nonalexithymic clients. Alexithymic clients that changed in alexithymia also changed in at least one of the mediators (lack of emotional awareness, emotional differentiation, or emotion regulation) of a previously proposed mediation model [44]. The model suggested that emotional awareness and emotional differentiation mediate the relationship between alexithymia and emotional regulation, defined as the ability to engage in healthy strategies to regulate emotions.

A recent systematic review and meta-analysis of 4 RCTs [45] showed that mindfulness-based interventions significantly reduce alexithymia, compared with a control condition, at the end of the study period. However, it was not clear how long the observed effect would last. Nevertheless, the findings indicate that mindfulness-based interventions may be an effective means of reducing alexithymia.

\section{Intervention Guidelines}

Considering the research on alexithymia and the outcome and process research on psychotherapy (e.g., [46]), some considerations can be made regarding better ways to address alexithymia in psychotherapy. The following suggested guidelines are based on the accumulated empirical research. First, the main findings are presented, and then, specific guidelines are recommended.

\section{Assess}

The idea of assessing alexithymia at the beginning of treatment is not new, especially in medical settings (e.g., [47]). Nevertheless, alexithymia has been under-recognized and underdiagnosed [48]. Assessing alexithymia at the beginning of a psychological treatment could help anticipate and prevent the challenges it brings to psychotherapies. From previous studies (e.g., [13, 49]), it seems not only valuable to assess alexithymic functioning but also to assess patients' understanding of that functioning and emotional experience. Alexithymia can be assessed by observation (e.g., Observer alexithymia Scale[OAS] [50]), with an interview (e.g., TSIA [23]), or with self-reports (e.g., TAS-20 $[24,51])$, for a full description of several measures see [22].
The use of self-report measures with alexithymic patients has been criticized. Nevertheless, the most widely studied and used measure of alexithymia is the TAS-20 $[51,52]$, a 20 -item self-report composed of 3 subscales: difficulty identifying feelings (DIF), difficulty describing feelings (DDF), and externally oriented thinking (EOT). The TAS-20 is adapted to different languages, and its factorial structural has been validated for different cultures. It has shown a stable and replicable factorial structure congruent with the alexithymia construct [52]. Accordingly, recent studies have criticized the use of subscales underlying that alexithymia is a construct and recommend that researchers and clinicians use a single total TAS-20 score and not subscale scores (e.g., [53]).

Some authors (e.g., [54]) have advocated that selfreporting for the EOT subscale may be more accurate than that of the other subscales. The EOT subscale does not require participants to judge their deficits regarding the experience and expression of emotions compared to the DIF and DDF subscales. In line with this idea, other researchers encourage a combination of the TAS with instruments that assess the ability to identify and express emotions to better understand clients' emotional experience (e.g., [55]) and help case conceptualization. The TSIA [23] has been considered an adequate measure to assess alexithymia. Since it is an interview, it allows addressing the content from the client's point of view.

Additionally, from the existing research, clinicians could anticipate some markers - emotional and relational - that could make the therapist anticipate s/he is working with a client with alexithymia. Some of these markers have been described, such as clients showing difficulty identifying and expressing their feelings, difficulties in identifying emotional markers on clients, difficulties in feeling close and connected with the client, and potential feelings of boredom from the therapist.

Guideline 1.1. Alexithymia should be routinely assessed either by self-report measures or clinically by considering markers.

Guideline 1.2. Clinical markers of alexithymia are difficulty in expressing, naming, and elaborating emotions.

Guideline 1.3. Difficulties in establishing a warm relationship and negative feelings (e.g., boredom or detachment) toward the client are clues for an alexithymic functioning.

\section{Case Conceptualization}

As previously mentioned, alexithymia has been associated with several different disorders. It should not be considered a disorder but a psychological characteristic of 
thought, feelings, and underlying processing $[17,34]$. So, it should not be considered independently of other characteristics of the client. Alexithymia has been conceptualized as a defense mechanism but also as a deficit pathology. It overlaps with the concept of mentalization (difficulty to take the perspective of others). Considering this, it should be considered both as a trait and a state phenomenon [56] since this construct encompasses features that may be stable and associated with personality functioning, as well as a transitory state associated with other difficulties (e.g., PTSD).

There is currently significant evidence, and is recognized the need to adapt or tailor psychotherapy to the individual (e.g., [57]). Furthermore, to present an alexithymic functioning in the context of neurodiversity, such as Asperger's syndrome, may be different than to present it in association with depression or a personality disorder. It is important to integrate that assessment in case conceptualization to guide intervention and adapt it to the individual's needs. Additionally, the different features of the construct seem to play an essential role in psychotherapy and may have a differential influence on the therapeutic alliance. Alexithymia influences the perspective the therapist has on the client. Therefore, even though it has been argued that alexithymia should be assessed as a global construct [53], specific features of alexithymia should be considered. Namely, DIF, DDF, and EOT may have different roles in psychotherapy intervention. In line with this, da Silva et al. [17] have suggested using the term alexithymia functioning to enhance the functional perspective of alexithymia rather than a static one.

Guideline 2.1. Alexithymia should be considered as a transdiagnostic dimension in case conceptualization.

Guideline 2.2. Alexithymia should be considered a description of emotional functioning (i.e., alexithymic functioning) rather than a type.

Guideline 2.3. Alexithymic functioning is particularly relevant in conceptualizing emotional processing and emotional aspects of the therapeutic relationship.

\section{Emotion Awareness and Regulation}

Some authors researching interventions aiming for the reduction of alexithymia have suggested the use of psychoeducation (e.g., [41, 43]). Several aspects have been identified to help the client build a coherent narrative and explore, even if cognitively, emotional dimensions. These aspects are developing a vocabulary for emotions, learning how to read others' emotions [41], and the relationship between alexithymia and early life experiences [43].

Guideline 3.1. Intervention should have a psychoeducational component concerning emotional identification and naming.
Alexithymia has been referred to as a disorder of emotion regulation [2]. However, the emotion regulation concept itself is complex and involves several emotional abilities (e.g., [58]). This highlights the importance of emotions in psychotherapy and the need to consider the differences between patients in their ability to process emotions when they come to treatment.

Taylor [12] suggested that psychotherapy involving specific techniques to increase emotional awareness and integrate symbolic elements of emotional schemes may be beneficial in reducing alexithymic characteristics. Changes in alexithymia seem associated with giving meaning to emotional experience, linking emotions to events, or gaining access to new perspectives (e.g., [13]). Meaning, not only being aware and tolerating emotions but also having a coherent emotional narrative.

Sensorial maps and images that constitute complex feelings incorporate facts about the interior of the organism. This informative role is one of the primary functions of feelings, among others. Feelings provide the will to behave accordingly to the situation [59]. Several interventions may help clients being more aware of their emotions and improve the distinction between bodily sensations (e.g., $[17,45])$. Among these interventions are focusing, techniques for awareness of sensation, mindfulnessbased interventions, or other experiential approaches.

In line with this, expressive writing [60] may be a helpful technique. It is useful to help clients assess their inner emotions, externalize them, and may also foster a sense of autonomy and control. This may be particularly beneficial for clients with alexithymia because they experience difficulties in interpersonal self-disclosure [61]. In a previous study of the effects of a novel psychological attribution and emotional awareness and expression therapy for chronic musculoskeletal pain [62], expressive writing was conducted in session and daily homework. It consisted of writing about stress and emotions in freewriting prose, unsent letters, and imagined dialogs. Even though this was not a study specifically aimed to target alexithymia, results showed a consistent reduction in alexithymia scores.

Guideline 3.2. Emotional regulation lies at the core of alexithymic functioning and should be given primacy in the intervention.

Guideline 3.3. Emotional awareness and elaboration are essential aspects of emotional regulation and should be addressed for sensitive issues.

Guideline 3.4. Experiential interventions may help increase awareness, while reflexive tasks may be helpful for emotional elaboration. 
The relational difficulties associated with the aforementioned emotional challenges may suggest the need for specific social and emotional learning (SEL) [63]. To acquire and effectively apply the knowledge, attitudes, and skills necessary to understand and manage emotions, set and achieve positive goals, feel and show empathy for others, establish and maintain positive relationships, and make conscientious decisions. Considering the Collaborative for Academic, Social, and Emotional Learning's (CASEL) Framework for Systemic Social and Emotional Learning may help enhance these emotional skills. CASEL is a unified framework that highlights 5 SEL competencies promoted through multiple contexts. SEL is the process through which children and adults develop the skills, knowledge, and attitudes necessary to understand and manage emotions, set and achieve positive goals, feel and show empathy for others, establish and maintain positive relationships, and make responsible decisions. The CASEL's model addresses 5 broad and inter-related areas of competence and highlights illustrative examples for each: self-awareness, self-management, social awareness, relationship skills, and responsible decision-making. According to the model, self-awareness is the ability to accurately recognize one's own emotions, thoughts, and values. Self-management regards the ability to successfully regulate one's emotions, thoughts, and behaviors in different situations. Social awareness is related to the ability to take the perspective of and empathize with others, including those from diverse backgrounds and cultures. Relationship skills encompass the ability to establish and maintain healthy and rewarding relationships with diverse individuals and groups. Moreover, responsible decision-making involves making constructive choices about personal behavior and social interactions based on ethical standards, safety concerns, and social norms.

The proposal is to use a SEL program integrated into psychotherapy. This would serve to develop the social and emotional skills lacking in alexithymic clients. This suggestion has not been explicitly tested for alexithymic clients. Nevertheless, it seems an interesting approach to the difficulties associated with alexithymia that may integrate all the aspects mentioned above. This could be done individually or in group intervention. One aspect that seems to arise from the studies on intervention is the impact group therapy seems to have on alexithymic clients [31]. For example, in a study on treatment preferences among psychiatric outpatients [64], patients who preferred group therapy had higher levels of alexithymia than those who preferred individual therapy. The authors suggested that clients with alexithymia may perceive group therapy as a setting that allows them to be more passive. On the other hand, group therapy provides an opportunity to learn how to work with feelings in relationships with others. Also, the group can potentiate social skills training and emotion sharing by vicarious learning.

The recommendation is that, similar to a Dialectical Behavior Therapy program [65], some of these interventions may be provided in group therapy: psychoeducation interventions, social skills training, and emotional regulation skills. Interestingly, this idea of integration is not new, considering that in 1988, Swiller [66] had discussed the opportunities that group therapy could provide to alexithymic clients.

Another alternative may be online-based interventions. Even though it could be necessary the social interactions given by presential individual or group psychotherapy, given that alexithymic individuals often display concern about relational interactions as experienced in traditional therapy, online interventions may be beneficial. In a pilot trial, individuals reporting high levels of alexithymia were randomly assigned to a blended smartphone-based intervention, including a psychoeducation session and 14 days of training with the mindtastic alexithymia app or a psychoeducation-only control condition. The study's findings provided preliminary evidence that smartphone-based interventions can improve emotion recognition skills in alexithymic individuals [67].

Guideline 3.5. Given the centrality of emotion and emotion expression in interpersonal relationships, intervention should include emotional and social skills training.

Guideline 3.6. This training should focus on the identification, elaboration, and expression of emotions in interpersonal contexts.

Guideline 3.7. Deeper emotional issues associated with intimacy should be addressed in more advanced stages of the intervention.

Guideline 3.8. When possible, group interventions should complement the individual intervention.

Guideline 3.9. When possible, integrating online interventions should complement intervention since it can be a good first step on treatment, especially concerning psychoeducational aspects.

\section{Therapeutic Alliance}

Ogrodniczuk [68] stressed the importance of identifying forms of therapeutic communication that effectively reduce alexithymic functioning so that psychotherapy can be more responsive to patients' needs. Given the importance of attachment theories on affect regulation, several studies pointed the relationship between attachment style and alexithymia (e.g., $[69,70])$, social deficits (e.g., $[71,72])$, and personality disorders (e.g., [9]). Consider- 
ing its interpersonal impact, Vanheule et al. [61] even argued that to make therapy for alexithymia-related disorders effective, a conceptualization of interpersonally imbedded affect regulation was needed.

Relationship building and emotional processing are related. Social psychologists and emotion theorists noted that expression plays a key role in structuring relationships (e.g., [70]). Research suggests that there are 3 possible mechanisms by which expression might alleviate distress, by focusing on the role of expression in (a) reducing distress about distress, (b) facilitating insight, and (c) affecting interpersonal relationships in a desired way [73]. For example, facial expressions serve as ongoing cues that influence social interactions, convey personal feelings to others, evoke subsequent emotional responses in others, and influence other people's behavior [74]. Da Silva et al. [36] compared alexithymic and nonalexithymic cases with good and poor outcomes. Both alexithymic cases and the poor outcome nonalexithymic showed difficulties in the therapeutic alliance, especially from the therapist's perspective. Emotional impairments such as being more overwhelmed or avoiding experiencing may have contributed to a poorer therapeutic alliance, impacting the outcome.

Ogrodniczuk et al. [34] observed that patients with alexithymia may have poorer outcomes in part because their therapists perceive them as having less value and less compatibility with them or they may be seen as less significant members when in group therapy. Therapists may unintentionally express these feelings, which may affect the patient's experience of therapy, contributing to poorer outcomes. In therapeutic interactions, these patients tend to focus on external events rather than on their mental states. In general, communicating about affective experiences is complex [61] and may evoke a communication interaction that promotes questioning from the other person [36]. Most therapists working with alexithymic patients refer feelings of boredom and a sense of less openness of the client, usually interacting with concise sentences or with yes/no answers. It is hypothesized that the patient uses projective identification to discharge unbearable psychic tension into the therapist, whose boredom is partly a defense against this (for a review see, [11]). Considering this, it might help think in terms of dysfunctional interpersonal cycles [75]. Recognizing the client's interpersonal cycle may help understand his/her functioning. For example, difficulties with describing their problems, expressing emotions, not getting the help they need, or evoking feelings of boredom in the therapist.

The expression of empathy from the therapist has an important role in the therapeutic process and its out- come. Empathy has an interpersonal function allowing the understanding of others, their goals, intentions, and feelings. Empathy also facilitates exploration, promotes openness to experience, and a curious attitude toward emotional experience, while enabling the deconstruction of assumptions and values. Empathy assists clients with self-reflection [76, 77], which may be extremely important with alexithymic patients since it can work as a corrective emotional experience. A corrective emotional experience requires protected circumstances, empathy, and awareness from the therapist. The corrective emotional experience is felt by the client, who expects specific responses from people, when s/he is surprised by the therapist's disconfirmation of the expected response. It is a tool available to the therapist who has formed a good therapeutic alliance with the patient $[78,79]$.

Guideline 4.1. Therapists should recognize the impact of alexithymic functioning (specifically the lack of emotional expression and warmth) on their feelings toward the client.

Guideline 4.2. Therapists should strive to provide an emotionally corrective experience through their relationship with the client.

Guideline 4.3. Therapists should use the relationship as a basis for emotional insight and model functional emotional expression and communication.

\section{Discussion}

From the different studies, several interventions may help clients with alexithymic functioning. Table 1 summarizes the suggested guidelines. A first important aspect for the psychotherapist is to be aware of this dimension. Only then can it be assessed and understood in line with the case conceptualization. Alexithymia should be considered as a transdiagnostic dimension in case conceptualization. Case conceptualization can help therapists understand how and why the client function the way he does and anticipate some obstacles and challenges that may arise in the intervention that should be seen as an intrinsic part of the therapeutic process. Some anticipated challenges concern emotional regulation deficits associated with emotional awareness difficulties. Also, most research points to challenges within the therapeutic alliance. These challenges may be related to the difficulties expressing feelings and the feelings the client may evoke on the therapist. Being aware of these aspects can help build the case conceptualization and acknowledging them for the benefit of the intervention. Even though it is essential to assess alexithymia as a complete construct, with its different components, it is also important, re- 
Table 1. Guidelines for intervention with alexithymic clients

\begin{tabular}{|c|c|}
\hline & Guideline \\
\hline Assess & $\begin{array}{l}\text { 1.1. Alexithymia should be routinely assessed either by self-report measures or clinically by considering } \\
\text { markers } \\
\text { 1.2. Clinical markers of alexithymia are difficulty in expressing, naming, and elaborating emotions } \\
\text { 1.3. Difficulties in establishing a warm relationship and negative feelings (e.g., boredom or detachment) } \\
\text { toward the client are clues for an alexithymic functioning }\end{array}$ \\
\hline $\begin{array}{l}\text { Emotion awareness and } \\
\text { regulation }\end{array}$ & $\begin{array}{l}\text { 3.1. Intervention should have a psychoeducational component concerning emotional identification and } \\
\text { naming } \\
\text { 3.2. Emotional regulation lies at the core of alexithymic functioning and should be given primacy in the } \\
\text { intervention } \\
\text { 3.3. Emotional awareness and elaboration are essential aspects of emotional regulation and should be } \\
\text { addressed for sensitive issues } \\
\text { 3.4. Experiential interventions may help increase awareness, while reflexive tasks may be helpful for } \\
\text { emotional elaboration } \\
\text { 3.5. Given the centrality of emotion and emotion expression in interpersonal relationships, intervention } \\
\text { should include emotional and social skills training } \\
\text { 3.6. This training should focus on the identification, elaboration, and expression of emotions in interpersonal } \\
\text { contexts } \\
\text { 3.7. Deeper emotional issues associated with intimacy should be addressed in more advanced stages of the } \\
\text { intervention } \\
\text { 3.8. When possible, group interventions should complement the individual intervention } \\
\text { 3.9. When possible, integrating online interventions should complement intervention since it can be a good } \\
\text { first step on treatment, especially concerning psychoeducational aspects }\end{array}$ \\
\hline
\end{tabular}

garding intervention, to acknowledge how each of those components affects treatment.

Assessing alexithymia, collecting relevant information for case conceptualization, exploring emotional states, enhancing emotional awareness, improving emotional regulation, and focusing on the therapeutic alliance may be central points in treatment. Integrating several strategies, the intensity of treatment, with different interventions such as group and individual therapy, to increase the outcome, may be a relevant approach to address the challenges associated with clients with alexithymic functioning.

This review was focused on the context of psychotherapy, trying to integrate the existing research up to this point. Alexithymia may impact other settings, but that was beyond the scope of this article. In the last years, there has been an effort to increase the knowledge regarding alexithymia and its specific impact on psychotherapy. Nevertheless, more awareness of the concept is needed to start being considered in case conceptualization for assisting intervention.

This work presents some limitations. An important point is that these guidelines take off from a limited number of studies, especially assessing and targeting alexithymia treatment. Another aspect may concern the lack of engagement or reservations about entering treatment. Even though it is not clear if this is a central aspect in alexithymia functioning, there are several people we may not reach or motivate for entering treatment. A possible way to enhance the client's adherence to psychotherapy could be related to online-based interventions - specifically using APPs. This could be a first step of treatment to capture clients and mo- 
tivate clients to a psychotherapeutic process. Nevertheless, we should consider that generally, there is also some attrition on these kinds of interventions (e.g., [80]).

A final reflection concerns the possibility of these guidelines being closer to specific theoretical orientations. Having this in mind, further studies are needed that analyze the impact of these proposals or develop a program with enough flexibility that allows integrating all these dimensions into intervention.

\section{Acknowledgment}

The author would like to thank David D. Neto (Instituto Universitário [ISPA]) and Applied Psychology Research Center Capabilities \& Inclusion [APPsyCI], Lisboa, Portugal) for his language editing and proofreading of the text.

\section{Conflict of Interest Statement}

The author declares no conflict of interest.

\section{Funding Sources}

The author received no external funding for this paper.

\section{Author Contributions}

A.N.S. did the literature review and wrote the manuscript.

\section{References}

1 Sifneos PE. The prevalence of "alexithymic" characteristics in psychosomatic patients. Psychother Psychosom. 1973;22(2):255.

2 Taylor GJ, Bagby RM, Parker JDA. Disorders of affect regulation: alexithymia in medical and psychiatric illness. UK: Cambridge University Press; 1999.

3 Merino H, Godás A, Pombo MG. Alexitimia y características psicológicas asociadas a actitudes alimentarias en una muestra de adolescentes. Rev de Psicopatol y Psicol Clin. 2002; 7(1):35-44.

4 Petterson R. Nameless desire: alexithymia and the anorexic patient. Am J Psychoanal. 2004;64: 77-90.

5 Uzun Ö, Ates A, Cansever A, Ozsahin A. Alexithymia in male alcoholics: study in a Turkish sample. Compr Psychiatry. 2003;44:349-52.

6 Li S, Zhang B, Guo Y, Zhang J. The association between alexithymia as assessed by the 20-item Toronto Alexithymia Scale and depression: a meta-analysis. Psychiatry Res. 2015;227:1-9.

7 Zlotnick C, Mattia JI, Zimmerman M. The relationship between posttraumatic stress disorder, childhood trauma and alexithymia in an outpatient sample. J Traum Stress. 2001;14(1): $177-88$.

8 Nicolò G, Semerari A, Lysaker PH, Dimaggio G, Conti L, D'Angerio S, et al. Alexithymia in personality disorders: correlations with symptoms and interpersonal functioning. Psychiatry Res. 2011;190:37-42.

9 De Panfilis C, Ossola P, Tonna M, Catania L, Marchesi C. Finding words for feelings: the relationship between personality disorders and alexithymia. Pers Individ Differ. 2015;74:28591.

10 Hill E, Berthoz S, Frith U. Brief report: cognitive processing of own emotions in individuals with autistic spectrum disorder and in their rel- atives. J Autism Dev Disord. 2004;34:229-35.

11 Taylor GJ. Alexithymia: concept, measurement, and implications for treatment. Am J Psychiatry. 1984;141:725-32.

12 Taylor GJ. Recent developments in alexithymia theory and research. Can J Psychiatry. 2000;45: $134-42$.

13 da Silva AN, Vasco AB, Watson JC. Alexithymia and emotional processing: a longitudinal mixed methods research. Res Psychother. 2018;21:40-54.

14 Bermond B, Vorst HC, Moormann PP. Cognitive neuropsychology of alexithymia: implications for personality typology. Cogn Neuropsychiatry. 2006;11:332-60.

15 Koelen JA, Eurelings-Bontekoe EH, Stuke F, Luyten P. Insecure attachment strategies are associated with cognitive alexithymia in patients with severe somatoform disorder. Int $J$ Psychiatry Med. 2015;49:264-78.

16 Schimmenti A, Caretti V. Attachment, trauma, and alexithymia. In: Luminet O, Bagby RM, Taylor GJ, editors. Alexithymia: advances in research, theory, and clinical practice. UK: Cambridge University Press; 2018. p. 127-41.

17 da Silva AN, Vasco AB, Watson JC. Alexithymia and emotional processing as phenomena pertinent to all theoretical orientations. Int J Psychother. 2013;17:5-19.

18 Šago D, Babić G. Roots of alexithymia. Arch Psychiatry Res. 2019;55(1):71-84.

19 Keefer KV, Taylor GJ, Parker JDA, Bagby RM. Taxometric analysis of the Toronto structured interview for alexithymia: further evidence that alexithymia is a dimensional construct. Assessment. 2019;26:364-74.

20 Parker JD, Keefer KV, Taylor GJ, Bagby RM. Latent structure of the alexithymia construct: a taxometric investigation. Psychol Assess. 2008; 20:385-96.
21 Taylor GJ, Bagby RM. Examining proposed changes to the conceptualization of the alexithymia construct: the way forward tilts to the past. Psychother Psychosom. 2020;3:1.

22 Sekely A, Bagby RM, Porcelli P. Assessment of the alexithymia construct. In: Luminet O, Bagby RM, Taylor GJ, editors. Alexithymia: advances in research, theory, and clinical practice. UK: Cambridge University Press; 2018. p. $17-$ 32.

23 Bagby RM, Taylor GJ, Parker JDA, Dickens SE. The development of the Toronto structured interview for alexithymia: item selection, factor structure, reliability and concurrent validity. Psychother Psychosom. 2006;75:25-39.

24 Bagby RM, Parker JDA, Taylor GJ. The twentyitem Toronto Alexithymia scale-I. Item selection and cross-validation of the factor structure. J Psychosom Res. 1994;38:23-32.

25 Todarello O, Taylor GJ, Parker JD, Fanelli M. Alexithymia in essential hypertensive and psychiatric outpatients: a comparative study. J Psychosom Res. 1995;39:987-94.

26 Wise TN, Mann LS, Hill B. Alexithymia and depressed mood in the psychiatric patient. Psychother Psychosom. 1990;54:26-31.

27 McCallum M, Piper WE, Ogrodniczuk JS, Joyce AS. Relationships among psychological mindedness, alexithymia and outcome in four forms of short-term psychotherapy. Psychol Psychother. 2003 Jun;76(2):133-44.

28 Leweke F, Bausch S, Leichsenring F, Walter B, Stingl M. Alexithymia as a predictor of outcome of psychodynamically oriented inpatient treatment. Psychother Res. 2009;19:323-31.

29 Krystal H. Alexithymia and psychotherapy. Am J Psychother. 1979;33:17-31.

30 Taylor GJ, Bagby RM. Psychoanalysis and empirical research: the example of alexithymia. J Am Psychoanal Assoc. 2013;61:99-133. 
31 Ogrodniczuk JS, Kealy D, Hadjipavlou GA, Cameron K. Therapeutic issues. In: Luminet $\mathrm{O}$, Bagby RM, Taylor GJ, editors. Alexithymia: advances in research, theory, and clinical practice. UK: Cambridge University Press; 2018. p. 190 206.

32 Cameron K, Ogrodniczuk J, Hadjipavlou G. Changes in alexithymia following psychological intervention: a review. Harv Rev Psychiatry. 2014;22:162-78

33 Ogrodniczuk JS, Piper WE, Joyce AS. Alexithymia as a predictor of residual symptoms in depressed patients who respond to short-term psychotherapy. Am J Psychother. 2004;58:15061.

34 Ogrodniczuk JS, Piper WE, Joyce AS. The negative effect of alexithymia on the outcome of group therapy for complicated grief: what role might the therapist play? Compr Psychiatry. 2005;46:206-13.

35 Quilty LC, Taylor GJ, McBride C, Bagby RM Relationships among alexithymia, therapeutic alliance, and psychotherapy outcome in major depressive disorder. Psychiatry Res. 2017;254: 75-9.

36 da Silva ACN, Vasco AB, Watson JC. Alexithymia and therapeutic alliance: a multiple case study comparing good and poor outcome cases. Res Psychother. 2018;21:83-97.

37 da Silva AN, Branco Vasco A, Watson JC. Alexithymia and change process: contributions of a phase model. Couns Psychother Res. 2020 20(4):703-14

38 Ogrodniczuk JS, Piper WE, Joyce AS. Effect of alexithymia on the process and outcome of psychotherapy: a programmatic review. Psychiatry Res. 2011;190:43-8.

39 Rufer M, Moergeli H, Moritz S, Drabe N, Weidt S. Alexithymia and non-treatment: an Internet based study of 312 people with chronic anxiety. Compr Psychiatry. 2014;55:179-87.

40 Grabe HJ, Frommer J, Ankerhold A, Ulrich C, Groger R, Franke GH, et al. Alexithymia and outcome in psychotherapy. Psychother Psychosom. 2008;77:189-94

41 Levant RF, Halter MJ, Hayden EW, Williams $\mathrm{CM}$. The efficacy of alexithymia reduction treatment: a Pilot Study. J Mens Stud. 2009; 17(1):75-84

42 Levant R, Williams C, Hayden E. Alexithymia reduction treatment (ART): a manual for a brief psycho-educational intervention for treating normative male alexithymia, group therapy format. [Unpublished manual]. Akron, $\mathrm{OH}$ University of Akron; 2008.

43 Kennedy M, Franklin J. Skills-based treatment for alexithymia: an exploratory case series. Behav Change. 2002;19(3):158-71.

44 da Silva AN, Vasco AB, Watson JC. Alexithymia and emotional processing: a mediation model. J Clin Psychol. 2017;73:1196-205.

45 Norman H, Marzano L, Coulson M, Oskis A Effects of mindfulness-based interventions on alexithymia: a systematic review. Evid Based Ment Health. 2019;22:36-43.

46 Norcross JC, Wampold BE. A new therapy for each patient: evidence-based relationships and responsiveness. J Clin Psychol. 2018;74:1889906.

47 Lumley MA, Neely LC, Burger AJ. The assessment of alexithymia in medical settings: implications for understanding and treating health problems. J Pers Assess. 2007;89:230-46.

48 Tacon A. Alexithymia: a challenge for mental health nursing practice. Aust NZJ Ment Health Nurs. 2001;10:229-35.

49 Dong S, Ju Q, Qiu T, Feng S. Intervention effect of group treatment on different types of alexithymia. Chin J Clin Psychol. 2017;25:304-9.

50 Haviland MG, Warren WL, Riggs ML. An observer scale to measure alexithymia. Psychosomatics. 2000;41:385-92.

51 Bagby RM, Taylor GJ, Parker JD. The twentyitem Toronto alexithymia scale-II. Convergent, discriminant, and concurrent validity. J Psychosom Res. 1994:38:33-40.

52 Parker JD, Taylor GJ, Bagby RM. The 20-item Toronto alexithymia scale. III. Reliability and factorial validity in a community population. J Psychosom Res. 2003;55:269-75.

53 Carnovale M, Taylor GJ, Parker JDA, Sanches M, Bagby RM. A bifactor analysis of the 20-item Toronto Alexithymia Scale: further support for a general alexithymia factor. Psychol Assess. $2021 \mathrm{Jul} ; 33(7): 619-28$.

54 Henry JD, Phillips LH, Maylor EA, Hosie J, Milne AB, Meyer C. A new conceptualization of alexithymia in the general adult population: implications for research involving older adults. J Psychosom Res. 2006;60:535-43.

55 Kooiman CG, Bolk JH, Rooijmans HG, Trijsburg RW. Alexithymia does not predict the persistence of medically unexplained physical symptoms. Psychosom Med. 2004;66:224-32.

56 de Haan HA, van der Palen J, Wijdeveld TG, Buitelaar JK, De Jong CA. Alexithymia in patients with substance use disorders: state or trait? Psychiatry Res. 2014;216:137-45.

57 Norcross JC, Wampold BE. Evidence-based therapy relationships: research conclusions and clinical practices. Psychotherapy. 2011;48: 98-102.

58 Gratz KL, Roemer L. Multidimensional assessment of emotion regulation and dysregulation: development, factor structure, and initial validation of the difficulties in emotion regulation scale. J Psychopathol Behav Assess. 2004;26(1): 41-54.

59 Damasio A. Feeling \& knowing: making minds conscious. Cogn Neurosci. 2021 Jan;12(2):65-6.

60 Pennebaker JW, Smyth JM. Opening up by writing it down how expressive writing improves health and eases emotional pain. 3rd ed. New York: Guilford Press; 2016.

61 Vanheule S, Verhaeghe P, Desmet M. In search of a framework for the treatment of alexithymia. Psychol Psychother. 2011;84:84-110.

62 Burger AJ, Lumley MA, Carty JN, Latsch DV, Thakur ER, Hyde-Nolan ME, et al. The effects of a novel psychological attribution and emotional awareness and expression therapy for chronic musculoskeletal pain: a preliminary, uncontrolled trial. J Psychosom Res. 2016;81: $1-8$.
63 Collaborative for Academic Social and Emotional Learning. What is SEL? Available from: http://www.casel.org/what-is-sel/.

64 Ogrodniczuk JS, Piper WE, Joyce AS, Abbass AA. Alexithymia and treatment preferences among psychiatric outpatients. Psychother Psychosom. 2009;78:383.

65 Linehan MM. DBT skills training manual. 2nd ed. New York: Guilford Press; 2015.

66 Swiller HI. Alexithymia: treatment utilizing combined individual and group psychotherapy. Int J Group Psychother. 1988;38:47-61.

67 Lukas CA, Trevisi Fuentes H, Berking M. Smartphone-based emotion recognition skills training for alexithymia: a randomized controlled pilot study. Internet Interv. 2019;17 100250-6.

68 Ogrodniczuk JS. Alexithymia: considerations for the psychotherapist. Psychother Bull. 2007; 42:4-7.

69 Pedrosa Gil F, Scheidt C, Hoeger D, Nickel M Relationship between attachment style, parental bonding and alexithymia in adults with somatoform disorders. Int J Psychiatr Med. 2008 38:437-51.

70 Troisi A, D’Argenio A, Peracchio F, Petti P. Insecure attachment and alexithymia in young men with mood symptoms. J Nerv Ment Dis. 2001;189:311-6.

71 Vanheule S, Desmet M, Meganck R, Bogaerts S Alexithymia and interpersonal problems. J Clin Psychol. 2007;63:109-17.

72 Vanheule S, Vandenbergen J, Verhaeghe P, Desmet M. Interpersonal problems in alexithymia: a study in three primary care groups. Psychol Psychother. 2010;83:351-62.

73 Kennedy-Moore E, Watson JC. How and when does emotional expression help? Rev Gen Psychol. 2001;5(3):187-212.

74 Keltner D, Kring AM. Emotion, social function, and psychopathology. Rev Gen Psychol. 1998;2(3):320-42.

75 Safran JD, Muran JC. Resolving therapeutic alliance ruptures: diversity and integration. J Clin Psychol. 2000;56:233-43.

76 Elliott R, Watson JC, Goldman RN, editors. Learning emotion-focused therapy: the process-experiential approach to change. Washington, DC: American Psychological Association; 2004.

77 Greenberg LS, Goldman RN, editors. Clinical handbook of emotion-focused therapy. Washington, DC: American Psychological Association; 2018.

78 Alexander F, French TM. Psychoanalytic therapy: principles and application. New York, NY: Ronald Press; 1946.

79 Castonguay LG, Hill CE. Transformation in psychotherapy: corrective experiences across cognitive behavioral, humanistic, and psychodynamic approaches. Washington, DC: American Psychological Association; 2012.

80 Melville KM, Casey LM, Kavanagh DJ. Dropout from Internet-based treatment for psychological disorders. Br J Clin Psychol. 2010;49: 455-71. 